\title{
G

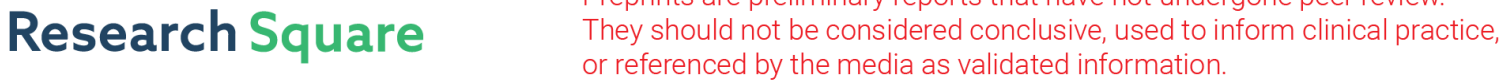

\section{A Multicenter Study of the Correlations of Anterior and Posterior Corneal Parameters in Chinese Myopic Patients}

\section{Yijun Hu}

Aier School of Ophthalmology, Central South University, Changsha

\section{Yanfang Wang}

National Key Discipline of Human Anatomy, School of Basic Medical Sciences, Southern Medical University, Guangzhou

\section{Shanqing Zhu}

Aier Institute of Refractive Surgery, Refractive Surgery Center, Guangzhou Aier Eye Hospital, Guangzhou

\section{Lu Xiong}

Aier Institute of Refractive Surgery, Refractive Surgery Center, Guangzhou Aier Eye Hospital, Guangzhou

\section{Xuejun Fang}

Refractive Surgery Center, Shenyang Aier Eye Hospital, Shenyang

\section{Jin Zhou}

Refractive Surgery Center, Chengdu Aier Eye Hospital, Chengdu

\section{Qingsong Zhang}

Refractive Surgery Center, Wuhan Aier Eye Hospital, Wuhan

\section{Xiaohua Lei}

Refractive Surgery Center, Hankou Aier Eye Hospital, Wuhan

\section{Yanbin Li}

National Key Discipline of Human Anatomy, School of Basic Medical Sciences, Southern Medical University, Guangzhou

\section{Wenhua Huang}

National Key Discipline of Human Anatomy, School of Basic Medical Sciences, Southern Medical University, Guangzhou

\section{Zheng Wang ( $\nabla$ gdwangzheng@163.com )}

Aier School of Ophthalmology, Central South University, Changsha

\section{Research Article}

Keywords: Anterior and Posterior, ophthalmic, eccentricity and asphericity

Posted Date: November 24th, 2020

DOI: https://doi.org/10.21203/rs.3.rs-109431/v1 
License: () (1) This work is licensed under a Creative Commons Attribution 4.0 International License. Read Full License 


\section{Abstract}

In this observational cross-sectional study consisting of 8365 Chinese myopic patients from five different ophthalmic centers, we investigated the correlations between anterior and posterior corneal parameters. Strongly negative anterior-posterior correlations of the mean corneal curvature were observed, with similar correlation coefficients in all myopic groups $(R:-0.85$ to -0.88$)$. The anterior-posterior correlations of corneal astigmatism ( $R: 0.65$ to 0.75$)$, eccentricity ( $R: 0.27$ to 0.38$)$ and asphericity $(R: 0.29$ to 0.41$)$ were all positive, with slightly different correlation coefficients between the myopic groups. The anterior-posterior correlations of mean corneal curvature were strongly negative with similar correlation coefficients in all quantile groups of six corneal and anterior chamber parameters ( $R$ : -0.84 to -0.91$)$, except $\operatorname{Sim~Km~}(R:-0.36$ to -0.64$)$. The anteriorposterior correlations of corneal astigmatism were all positive with slightly different correlation coefficients between the quantile groups of $\operatorname{SimKm}(R: 0.66$ to 0.74$)$. The anterior-posterior correlations of corneal eccentricity ( $R: 0.30$ to 0.44$)$ and asphericity $(R: 0.33$ to 0.45$)$ were positive and slightly different between the quantile groups of SimKm, pachy apex and corneal volume. In conclusion, anterior-posterior correlations of corneal curvature, astigmatism, eccentricity and asphericity can be affected by the severity of myopia and some other corneal parameters.

\section{Introduction}

It is commonly believed that the shape of the anterior and posterior corneal surfaces is highly correlated with each other. Previous studies have demonstrated moderate to strong correlations in parameters of the anterior and posterior corneal surfaces (e.g. corneal astigmatism, corneal curvature and corneal asphericity) in normal human eyes ${ }^{1-5}$ and in eyes with keratoconus. ${ }^{5-7}$ In fact, traditional methods of calculating total corneal astigmatism, known as keratometric astigmatism, are mainly based on measurement of the anterior corneal astigmatism (ACA), assuming the cornea a single dioptric surface with a fixed anterior to posterior curvature ratio. ${ }^{8}$ However, the anterior and posterior corneal surfaces are not always correlated according to some fixed ratios. ${ }^{9}$ This is particularly true in eyes with subclinical or early keratoconus, ${ }^{4,5}$ where changes at the posterior cornea proceed the anterior cornea. ${ }^{10}$ On the other hand, it is possible that the correlations between the two corneal surfaces vary according to different refractive status, corneal size and even depth of the anterior chamber. However, to date there is no study to verify this hypothesis.

Pentacam is a Scheimpflug-based imaging device for anterior segment structures including the cornea, anterior chamber, and lens. Using a rotating Scheimpflug camera, the device is capable of capturing 50 rotational images of the cross-sections of the anterior segment within 2 seconds. Based on the measurements, parameters of the anterior and posterior corneas and the anterior chamber are generated by a built-in software. Pentacam has been commonly used to measure both the anterior and posterior corneas due to the good repeatability and reproducibility. ${ }^{8}$ Simultaneous measurement of both the anterior and posterior corneal surfaces makes Pentacam an ideal device to study the correlations between the two corneal surfaces.

In the present study, we aim to investigate the anterior-posterior correlations of the corneal parameters according to different refractive status and anterior segment dimension in a large number of Chinese myopic patients from multiple ophthalmic centers. 


\section{Results}

A total of 8365 patients (8365 eyes) were recruited from five ophthalmic centers, including 2460 eyes from Guangzhou Aier Eye Hospital (GZ), 2435 eyes from Shenyang Aier Eye Hospital (SY), 1586 eyes from Chengdu Aier Eye Hospital (CD), 1556 eyes from Wuhan Aier Eye Hospital (WH), and 328 eyes from Hankou Aier Eye Hospital (HK). Mean age of the patients was $25.1 \pm 5.4$ years. Mean SE of the eyes was $-5.13 \pm 2.05 \mathrm{D}$. There were significant differences in age, gender and spherical equivalent (SE) among patients from different ophthalmic centers (all $P<0.001$ ). Demographics and corneal parameters of the eyes in the five ophthalmic centers are shown in Table 1.

Correlations of the anterior and posterior mean corneal curvature $(\mathrm{Km})$ were significantly negative with similar $\mathrm{R}$ values (-0.85 to -0.88$)$ in all the myopic groups (Figure $1 \mathrm{~A})$. On the contrary, correlations between the ACA and posterior corneal astigmatism (PCA) were significantly positive with slightly increased R values (0.65 to 0.75$)$ from the low myopia (LM) group to the extremely high myopia (EHM) group (Figure 1B). The eccentricity of anterior and posterior corneal surfaces was also correlated positively in all the myopic groups, while the LM, moderate myopia (MM) and high myopia (HM) groups presented slightly greater $\mathrm{R}$ values (0.36 to 0.38$)$ than the EHM group (0.27) (Figure 1C), and similar findings were observed in the asphericity of the anterior and posterior corneal surfaces (Figure 1D).

Correlations between the anterior and posterior $\mathrm{Km}$ were significantly negative in every quantile group (Q1-Q4), with lower R values observed in group Q1 (-0.63) and Q4 (-0.64) than in group of Q2 (-0.36) and Q3 (-0.37) SimKm (Figure 2A, Figure 3A), and with similar R values in quantile groups of pachy apex (PA, -0.90 to -0.91 ), corneal volume (CV, -0.90 to -0.91), anterior chamber height ( $\mathrm{ACH},-0.88$ to -0.89$)$, anterior chamber volume (ACV, -0.87 to -0.88 ), and white-to-white diameter (WTW, -0.84 to -0.85) (Figure 2A, Figure 3B-F).

Significant positive correlations between the ACA and PCA were observed in all the quantile groups, with the R values slightly increased with quantile levels of $\operatorname{SimKm}(0.66$ to 0.74$)$ (Figure 2B, Figure 4A), and with similar $R$ values in different quantile groups of PA (0.69 to 0.71), CV (0.68 to 0.72), ACH (0.70 to 0.72), ACV (0.69 to 0.71$)$ and WTW (0.69 to 0.72) (Figure 2B, Figure 4B-F).

Significant positive correlations were observed between the anterior and posterior corneal eccentricity in all the quantile groups, with the $\mathrm{R}$ values slightly increased with quantile levels of SimKm (0.31 to 0.43$)$, but slightly increased with quantile levels of PA (0.44 to 0.32) and CV (0.43 to 0.30) (Figure 2C, Figure 5A-C). The R values were similar across the quantile levels of $\mathrm{ACH}(0.31$ to 0.38$)$, ACV (0.32 to 0.36$)$ and WTW (0.34 to 0.37) (Figure 2C, Figure 5D-F).

Positive correlations were also observed between the anterior and posterior corneal asphericity in all the quantile groups. The $\mathrm{R}$ values were increased slightly increased with quantile levels of SimKm (0.34 to 0.45), but slightly increased with quantile levels of PA (0.45 to 0.36) and CV (0.44 to 0.34) (Figure 2D, Figure 6A-C). Similar with the correlations of eccentricity, the $\mathrm{R}$ values for asphericity correlation were similar across the quantile levels of $\mathrm{ACH}(0.33$ to 0.40$)$, ACV (0.33 to 0.39) and WTW (0.36 to 0.40) (Figure 2D, Figure 6D-F).

\section{Discussion}


In the present multicenter study, we demonstrated the correlations between the anterior and posterior corneal surfaces according to different refractive status and anterior segment dimension in a large number of Chinese myopic patients from different parts of mainland China (GZ from the south, SY from the northeast, CD from the southwest, WH and HK from the central). Using the pooled data of the five cohorts with diversity in age, sex and $\mathrm{SE}$, negative correlations were observed between the anterior and posterior $\mathrm{Km}$ while positive correlations were observed between the ACA and PCA, between anterior and posterior eccentricity, asl well as between anterior and posterior asphericity. The results were consistent with some previous studies. ${ }^{5,6}$ We also found that $R$ values of the anterior-posterior corneal correlations varied in different quantile groups of myopia and some corneal parameters (ie, SimKm, PA and CV), but not affected by the quantile levels of WTW and anterior chamber parameters (ie, $\mathrm{ACH}$ and $\mathrm{ACV}$ ).

Assessment of the corneal curvature plays an important role in refractive surgery planning, intraocular lens power calculation and early diagnosis of keratoconus ${ }^{6,11-13}$. In traditional keratometry, the cornea is assumed to have a fixed ratio between the anterior and poster corneal curvature, and the anterior corneal curvature is used to calculate the total corneal curvature. With recognition on the changes of anterior and posterior corneal curvature correlations, the ratio between the anterior and posterior curvature radii were adopted to evaluate the risk of hyperopia shift after corneal surgery. ${ }^{14}$ In the present study, the correlations between the anterior and posterior $\mathrm{Km}$ were similar in different myopic groups and quantile groups of PA, CV, ACH, ACV and WTW (Figures 1A, 2A and 3B-F). However, greater $R$ values between the anterior and posterior $\mathrm{Km}$ were observed in group Q2 and Q3 than in group Q1 and Q4 of SimKm (Figures 2A and 3A). This finding suggests that the correlations between the anterior and posterior $\mathrm{Km}$ are different in corneal with different refractive power, and that measuring both the anterior and posterior corneal curvatures is needed to obtain an accurate total corneal curvature. In the present study, the strength of the correlations between the anterior and posterior $\mathrm{Km}$ was strong, with $\mathrm{R}$ values of at least -0.85 in all the myopia groups. This result was consistent with a previous study in which the $R$ value was -0.94 for normal eyes and -0.85 for subclinical keratoconus, but the $R$ value was decreased in eyes with confirmed keratoconus ${ }^{5}$. Our result also indicated that the increase of myopia severity didn't result in reduced correlation strength between the anterior and posterior corneal curvatures.

Management of PCA during astigmatism correction has become a hot topic in cataract and refractive surgery, and many efforts have been made to predict the PCA using ACA. ${ }^{2,15}$ However, PCA prediction based on ACA alone is not accurate enough. ${ }^{9}$ In the present study, the R values for the correlation between ACA and PCA were increased from 0.65 in the LM group to 0.75 in the EHM group, indicating a stronger ACA-PCA correlation in eyes with higher severity of myopia. The R values of ACA-PCA correlation were also slightly increased with quantile levels of SimKm ( 0.66 to 0.74 ). These findings indicate that the ACA-PCA correlation is affected by multiple factors and the integration of various anterior segment parameters is needed to make a more accurate PCA prediction. ${ }^{1}$ Moreover, the moderate ACA-PCA correlations may be due to the greater hereditability of the posterior corneal surface compared to the anterior corneal surface ${ }^{16,17}$. Surprisingly, the strength of ACA-PCA correlation seems to be higher in keratoconus eyes compared to normal eyes, although controversy still remains. ${ }^{5-7}$ This may be due to the unsynchronized changes of the ACA and PCA during the development of keratoconus, and the tendency towards a better alignment of the PCA to the ACA in keratoconus eyes. ${ }^{16-18}$ 
In the present study, there were weak correlations (R: 0.29 to 0.45 ) between the anterior and posterior corneal asphericity in all the quantile groups. These findings were different from previous studies where poor anteriorposterior corneal asphericity correlations in normal eyes ${ }^{1,5}$ and good correlations in keratoconus eyes were observed. ${ }^{1,2}$ It was shown that the $\mathrm{R}$ values for the anterior-posterior corneal asphericity correlations in normal eyes were $0.03-0.04$ and 0.17 in the previous studies. ${ }^{1,5}$ On the contrary, the $\mathrm{R}$ values of the correlations were 0.62-0.89 and 0.86-0.94 in keratoconus eyes. ${ }^{1,2}$ These findings together suggest that in terms of the anteriorposterior corneal asphericity correlation, myopic eyes are in between the normal eyes and keratoconus eyes. The roles of anterior-posterior corneal asphericity correlations in corneal ectasia warrants further investigation. Like the ACA-PCA correlations, the anterior-posterior corneal asphericity correlations are also affected by multiple factors such as myopia severity, SimKm, PA, and CV, suggesting complex interactions between the anterior-posterior corneal shapes and other anterior segment parameters.

In the present study, the $\mathrm{R}$ values for anterior-posterior correlations of corneal astigmatism, eccentricity and asphericity were increased with higher quantile levels of SimKm, but the $\mathrm{R}$ values for anterior-posterior correlations of corneal eccentricity and asphericity were decreased with higher quantile levels of PA and CV. These findings suggest that different the corneal parameters have different effects on the anterior-posterior corneal correlations. Further studies are required to investigate the mechanisms of each corneal parameter affecting the anterior-posterior correlations of corneal shapes.

Our study has some limitations. Firstly, the conclusions of our study can only be applied to myopic patients from the same age group. In older patients, the anterior-posterior corneal correlations may be different. Factors affecting these correlations may also be different in older patients. Secondly, the exact mechanisms underlying the anterior-posterior corneal correlations remain a mystery to us, although some affecting factors are identified in the present study. Thirdly, we didn't compare the anterior-posterior corneal correlations using data obtained from different imaging devices. Thus, we are unable to know whether our results can be used interchangeably with those obtained from other devices. Lastly, due to the cross-sectional design of the study we did not investigate the changes of anterior-posterior corneal correlations after corneal refractive surgery. Further studies are needed to address this issue.

In conclusion, the present study has demonstrated that the anterior-posterior correlations of corneal curvature, astigmatism, eccentricity and asphericity can be affected by the severity of myopia and some other corneal parameters.

\section{Methods}

\section{Participants}

This is a multicenter study involving five ophthalmic centers, including GZ (113.2 E $23.1 \mathrm{~N}$, altitude $43.4 \mathrm{~m})$, SY (123.4 E 41.8 N, altitude 51.0m), CD (104.0 E $30.7 \mathrm{~N}$, altitude 505.9m), WH (114.2 E $30.4 \mathrm{~N}$, altitude 23.3m), and HK (114.1 E 30.4 N, altitude 27.6m). ${ }^{19}$ The study has been approved by the Institutional Review Board (IRB) of Guangzhou Aier Eye Hospital, and is in agreement with the Declaration of Helsinki. Inclusion criteria and exclusion criteria were described previously. ${ }^{19}$ We only included the right eye from each patient for analysis. 
Because we only reviewed the patients' medical records, and no individual patient could be identified from the data, informed consent was waived by the IRB. ${ }^{19}$

\section{Examinations}

The examinations were previously described. ${ }^{19}$ All the eyes underwent routine preoperative examinations including best-corrected visual acuity (BCVA), intraocular pressure (IOP), cycloplegic and manifest refraction, anterior segment examination by slit-lamp, corneal topography and tomography measurements. The eyes were divided into four groups according to the manifest SE: LM (-3.00 D<SE $\leq-0.50 \mathrm{D}), \mathrm{MM}(-6.00 \mathrm{D}<\mathrm{SE} \leq-3.00 \mathrm{D})$, HM (-10.00 D<SE $\leq-6.00 \mathrm{D})$ and EHM (SE $\leq-10.00 \mathrm{D})$.

Pentacam examination was performed by experienced technicians. Operating of the Pentacam instrument (Pentacam HR, Oculus GmbH, Wetzlar, Germany) was described previously. ${ }^{19}$ Only scans covering at least central $8.0 \mathrm{~mm}$ of corneal surface with the image quality labelled as 'OK' on the display were accepted. The anterior and posterior corneal radius in the flattest and steepest meridians in the $3.0 \mathrm{~mm}$ central zone, eccentricity and asphericity of the anterior and posterior cornea, PA, CV in the $3.0 \mathrm{~mm}$ diameter area, WTW, ACH, and ACV were generated by the Pentacam instrument. The SimKm, ACA and PCA in the 3.0 mm central zone were calculated as previously described. ${ }^{20}$ Pentacam data of the eyes were retrieved from the machine and only results with image quality labelled with 'OK' were included. ${ }^{19}$

\section{Statistical Analysis}

The data from the five ophthalmic centers were pooled together for analysis. A Kolmogorov-Smirnov (KS) test was used to evaluate normality of all variables. Data of patient demographics were presented as mean \pm standard deviation (SD). Spearman correlation test was used for anterior-posterior correlation of mean corneal curvature, corneal astigmatism, corneal eccentricity and corneal asphericity, according to different myopic groups and the quantile groups of the corneal and anterior chamber parameters (SimKm, PA, CV, WTW, ACH and ACV)). $P<0.05$ was considered as statistically significant.

\section{Declarations}

\section{Data Availability Statement}

The data used during the current study are available from the corresponding author on reasonable request.

\section{Acknowledgements}

This work was supported by Grant 2018 SK50106 from the Technology Innovation Guidance Program of Hunan Province (Y.H.), Grant AR1909D2, AM1909D2 from the Science Research Foundation of Aier Eye Hospital Group (Y.H.), Grant 2018B090944002 from the Key Research and Development Program of Guangdong Province and Grant WX19C12 from the Medical Research Funding of Health Commission of Wuhan City (X.L.).

\section{Author Contributions}

Y.H., Y.W. and S.Z. designed the study, analyzed the data and wrote the manuscript. L.X, X.F, J.Z., Q.Z., and X.L. collected the data. Y.L. revised the manuscript. W.H. and Z.W. supervised the study and edited the manuscript. 
Competing financial interests: The authors declare no competing interests.

\section{References}

1. Montalban R, Pinero DP, Javaloy J, Alio JL. Scheimpflug photography-based clinical characterization of the correlation of the corneal shape between the anterior and posterior corneal surfaces in the normal human eye. Journal of cataract and refractive surgery. 2012;38(11):1925-1933.

2. Montalban R, Pinero DP, Javaloy J, Alio JL. Correlation of the corneal toricity between anterior and posterior corneal surfaces in the normal human eye. Cornea. 2013;32(6):791-798.

3. Dubbelman M, Sicam VA, Van der Heijde GL. The shape of the anterior and posterior surface of the aging human cornea. Vision research. 2006;46(6-7):993-1001.

4. Schlegel Z, Hoang-Xuan T, Gatinel D. Comparison of and correlation between anterior and posterior corneal elevation maps in normal eyes and keratoconus-suspect eyes. Journal of cataract and refractive surgery. 2008;34(5):789-795.

5. Pinero DP, Alio JL, Aleson A, Escaf Vergara M, Miranda M. Corneal volume, pachymetry, and correlation of anterior and posterior corneal shape in subclinical and different stages of clinical keratoconus. Journal of cataract and refractive surgery. 2010;36(5):814-825.

6. Tomidokoro A, Oshika T, Amano S, Higaki S, Maeda N, Miyata K. Changes in anterior and posterior corneal curvatures in keratoconus. Ophthalmology. 2000;107(7):1328-1332.

7. Montalban R, Alio JL, Javaloy J, Pinero DP. Correlation of anterior and posterior corneal shape in keratoconus. Cornea. 2013;32(7):916-921.

8. Camps VJ, Pinero Llorens DP, de Fez D, et al. Algorithm for correcting the keratometric estimation error in normal eyes. Optometry and vision science : official publication of the American Academy of Optometry. 2012;89(2):221-228.

9. Koch DD, Ali SF, Weikert MP, Shirayama M, Jenkins R, Wang L. Contribution of posterior corneal astigmatism to total corneal astigmatism. Journal of cataract and refractive surgery. 2012;38(12):20802087.

10. Golan O, Hwang ES, Lang P, et al. Differences in Posterior Corneal Features Between Normal Corneas and Subclinical Keratoconus. Journal of refractive surgery (Thorofare, NJ : 1995). 2018;34(10):664-670.

11. Seitz B, Langenbucher A, Nguyen NX, Kus MM, Küchle M. Underestimation of intraocular lens power for cataract surgery after myopic photorefractive keratectomy 11 The authors have no proprietary interest in the development or marketing of this or any competing instrument or piece of equipment. Ophthalmology. 1999;106(4):693-702.

12. Kim M, Eom Y, Lee H, Suh Y-W, Song JS, Kim HM. Use of the Posterior/Anterior Corneal Curvature Radii Ratio to Improve the Accuracy of Intraocular Lens Power Calculation: Eom's Adjustment Method. Investigative Ophthalmology \& Visual Science. 2018;59(2):1016-1024.

13. Morishige N, Magome K, Ueno A, Matsui T-A, Nishida T. Relations Among Corneal Curvature, Thickness, and Volume in Keratoconus as Evaluated by Anterior Segment-Optical Coherence Tomography. Investigative Ophthalmology \& Visual Science. 2019;60(12):3794-3802. 
14. Diener R, Eter N, Alnawaiseh M. Using the posterior to anterior corneal curvature radii ratio to minimize the risk of a postoperative hyperopic shift after Descemet membrane endothelial keratoplasty. Graefe's Archive for Clinical and Experimental Ophthalmology. 2020;258(5):1065-1071.

15. Miyake T, Shimizu K, Kamiya K. Distribution of posterior corneal astigmatism according to axis orientation of anterior corneal astigmatism. PloS one. 2015;10(1):e0117194.

16. Mahroo OA, Oomerjee M, Williams KM, O'Brart DPS, Hammond CJ. High Heritability of Posterior Corneal Tomography, as Measured by Scheimpflug Imaging, in a Twin Study. Investigative Ophthalmology \& Visual Science. 2014;55(12):8359-8364.

17. Heydarian S, Hashemi H, Yekta A, et al. Heritability of Corneal Curvature and Pentacam Topometric Indices: A Population-Based Study. Eye \& Contact Lens. 2019;45 (6):365-371.

18. Savini G, Næser K, Schiano-Lomoriello D, Mularoni A. Influence of Posterior Corneal Astigmatism on Total Corneal Astigmatism in Eyes With Keratoconus. Cornea. 2016;35(11):1427-1433.

19. Hu Y, Zhu S, Xiong L, et al. A multicenter study of the distribution pattern of posterior corneal astigmatism in Chinese myopic patients having corneal refractive surgery. Scientific reports. 2020;10(1):16151.

20. Næser K, Savini G, Bregnhøj JF. Corneal powers measured with a rotating Scheimpflug camera. The British journal of ophthalmology. 2016;100(9):1196-1200.

\section{Tables}

Table1. Demographics of the patients in the five ophthalmic centers.

\begin{tabular}{|c|c|c|c|c|c|c|c|}
\hline \multirow[t]{2}{*}{ Demographics } & \multicolumn{6}{|c|}{ Ophthalmic center } & \multirow{2}{*}{$\begin{array}{l}P \\
\text { Value* }\end{array}$} \\
\hline & GZ & SY & $C D$ & WH & HK & Pooled & \\
\hline Number & 2460 & 2435 & 1586 & 1556 & 328 & 8365 & - \\
\hline \multicolumn{8}{|l|}{ Age (years) } \\
\hline Mean $\pm S D$ & $26.9 \pm 5.4$ & $23.9 \pm 5.1$ & $24.2 \pm 5.5$ & $25.4 \pm 5.0$ & $23.9 \pm 4.8$ & $25.1 \pm 5.4$ & $<0.001$ \\
\hline Range & $18-40$ & $18-40$ & $18-40$ & $18-40$ & $18-38$ & $18-40$ & - \\
\hline \multicolumn{8}{|l|}{ Gender } \\
\hline Female $(n, \%)$ & $\begin{array}{l}1325 \\
(53.9 \%)\end{array}$ & $\begin{array}{l}887 \\
(36.4 \%)\end{array}$ & $\begin{array}{l}612 \\
(38.6 \%)\end{array}$ & $\begin{array}{l}770 \\
(49.5 \%)\end{array}$ & $\begin{array}{l}118 \\
(36.0 \%)\end{array}$ & $\begin{array}{l}3712 \\
(44.4 \%)\end{array}$ & $<0.001$ \\
\hline Male (n, \%) & $\begin{array}{l}1135 \\
(46.1 \%)\end{array}$ & $\begin{array}{l}1548 \\
(63.6 \%)\end{array}$ & $\begin{array}{l}974 \\
(61.4 \%)\end{array}$ & $\begin{array}{l}786 \\
(50.5 \%)\end{array}$ & $\begin{array}{l}210 \\
(64.0 \%)\end{array}$ & $\begin{array}{l}4653 \\
(55.6 \%)\end{array}$ & $<0.001$ \\
\hline \multicolumn{8}{|l|}{ SE (D) } \\
\hline Mean $\pm S D$ & $-5.18 \pm 2.2$ & $-4.84 \pm 1.70$ & $-5.26 \pm 2.23$ & $-5.28 \pm 1.92$ & $-5.64 \pm 2.64$ & $-5.13 \pm 2.05$ & $<0.001$ \\
\hline Range & $\begin{array}{l}-22.50 \text { to } \\
-0.63\end{array}$ & $\begin{array}{l}-11.25 \text { to } \\
-0.75\end{array}$ & $\begin{array}{l}-26.25 \text { to } \\
-1.00\end{array}$ & $\begin{array}{l}-20.38 \text { to } \\
-0.50\end{array}$ & $\begin{array}{l}-22.00 \text { t o- } \\
0.63\end{array}$ & $\begin{array}{l}-26.25 \text { to } \\
-0.50\end{array}$ & - \\
\hline
\end{tabular}

*Comparison among the five ophthalmic centers using Kruskal-Wallis test; SD, standard deviation; SE, spherical equivalent; D, diopter; GZ, Guangzhou Aier Eye Hospital; SY, Shenyang Aier Eye Hospital; CD, Chengdu Aier Eye 


\section{Figures}
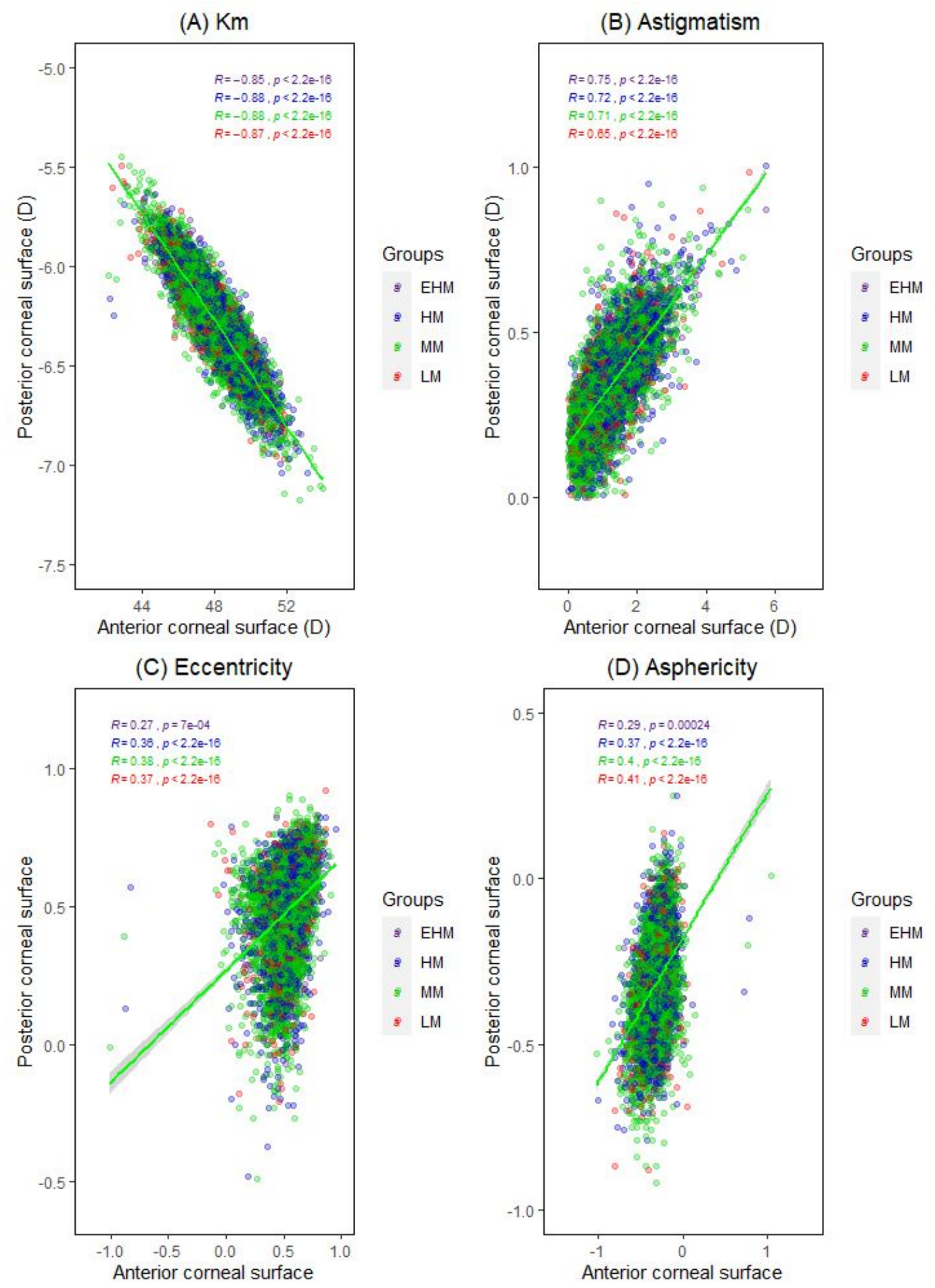

\section{Figure 1}

Scattergrams showing anterior-posterior correlations of mean corneal curvature (A), corneal astigmatism (B), corneal eccentricity (C) and corneal asphericity (D) in different myopic groups. 
(A) $\mathrm{Km}$

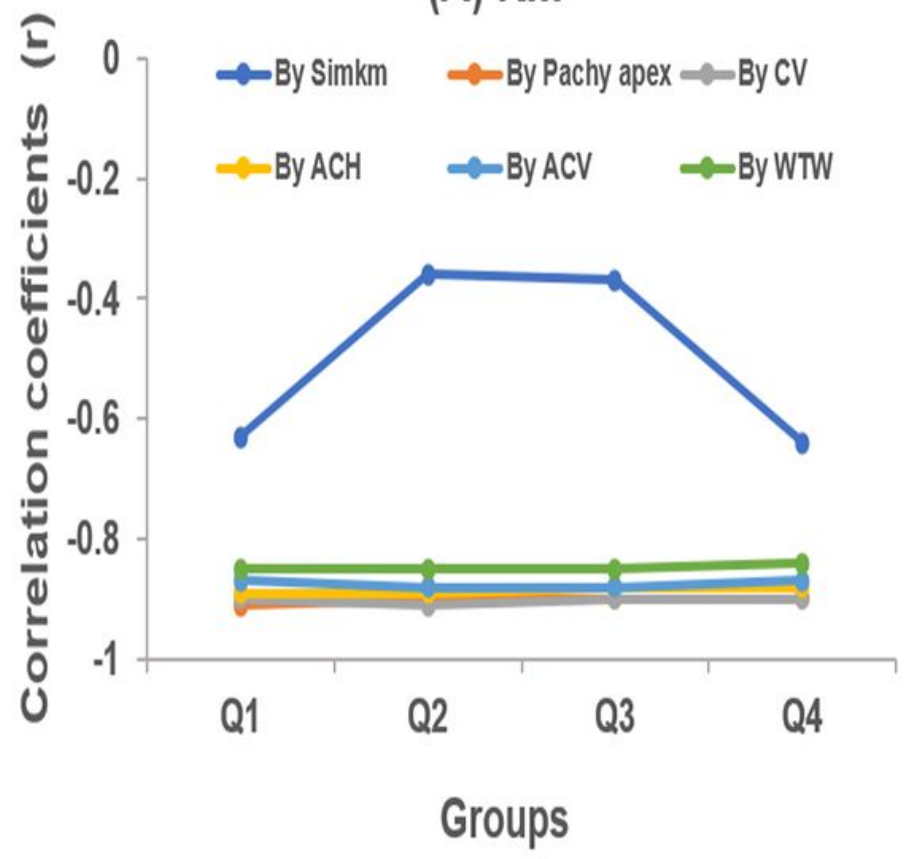

(C) Eccentricity

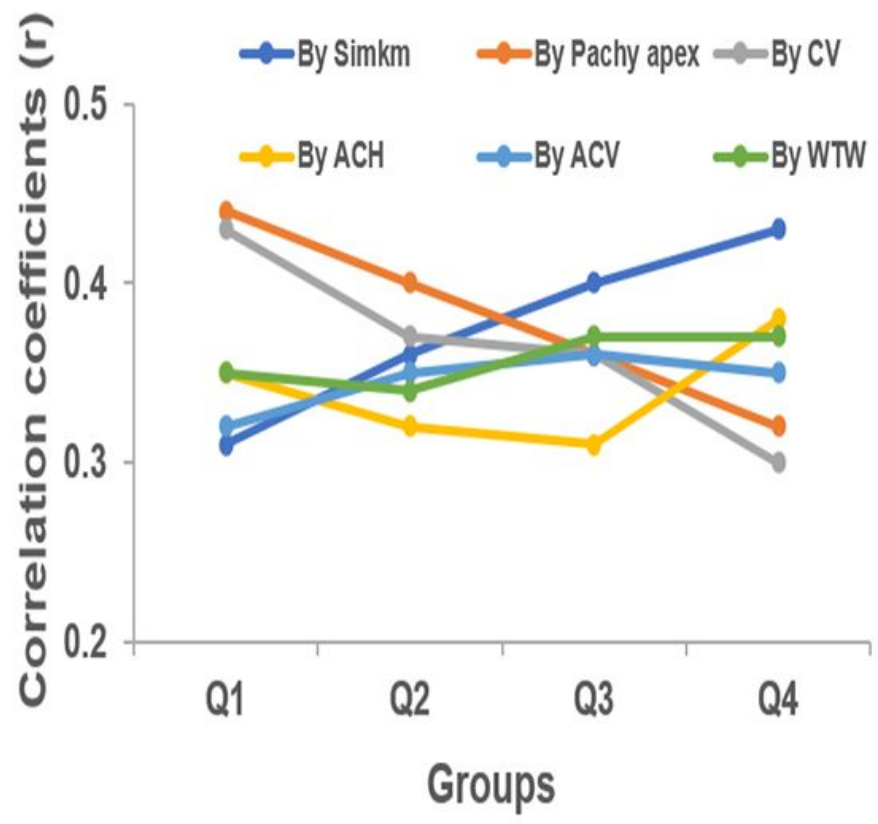

(B) Astigmatism

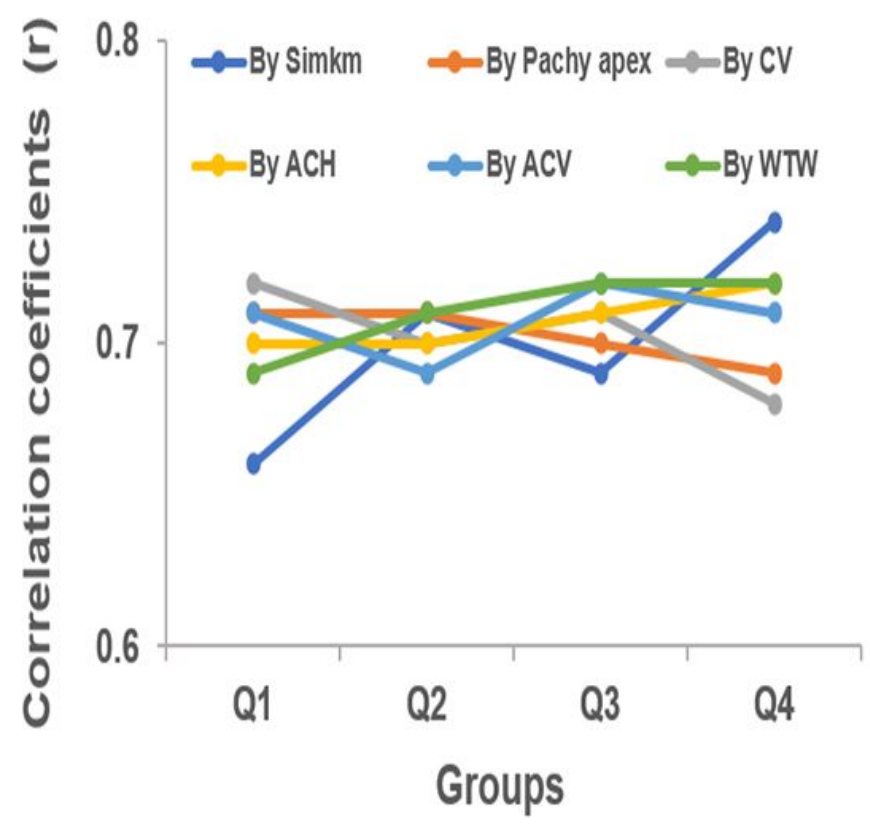

(D) Asphericity

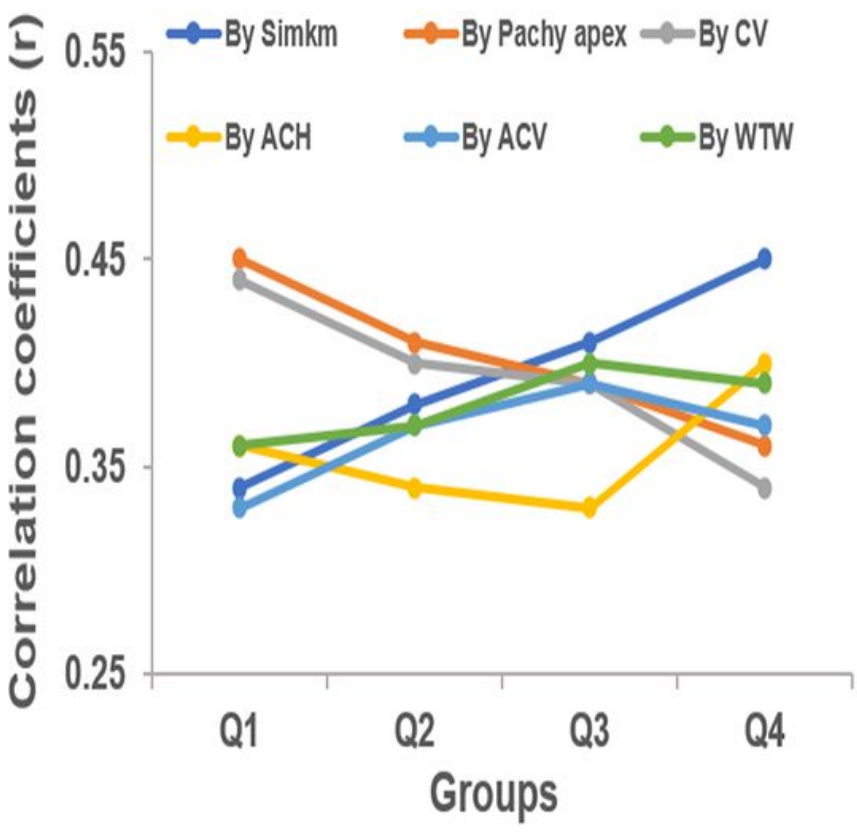

Figure 2

Spearman correlation coefficients of anterior-posterior correlations of mean corneal curvature (A), corneal astigmatism (B), corneal eccentricity (C) and corneal asphericity (D) in different quantile groups of SimKm, pachy apex, corneal volume (CV), anterior chamber height and volume (ACH and ACV), and white-to-white (WTW) diameter. 
(A) By SimKm

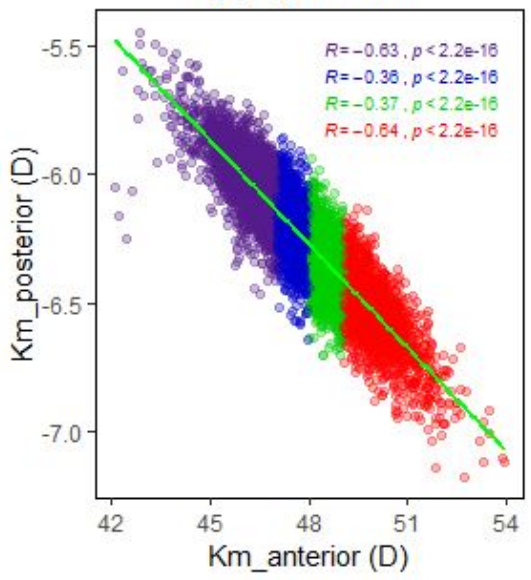

(C) By CV

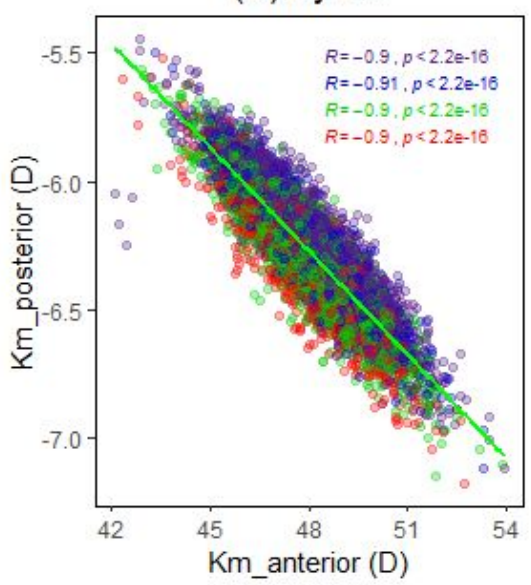

(E) By ACV

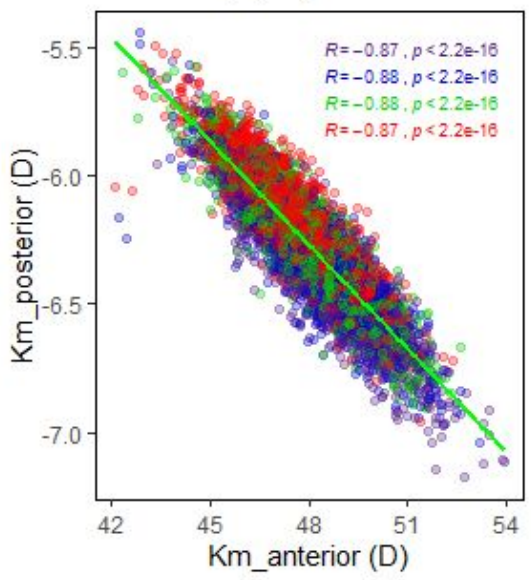

(B) By Pachy apex

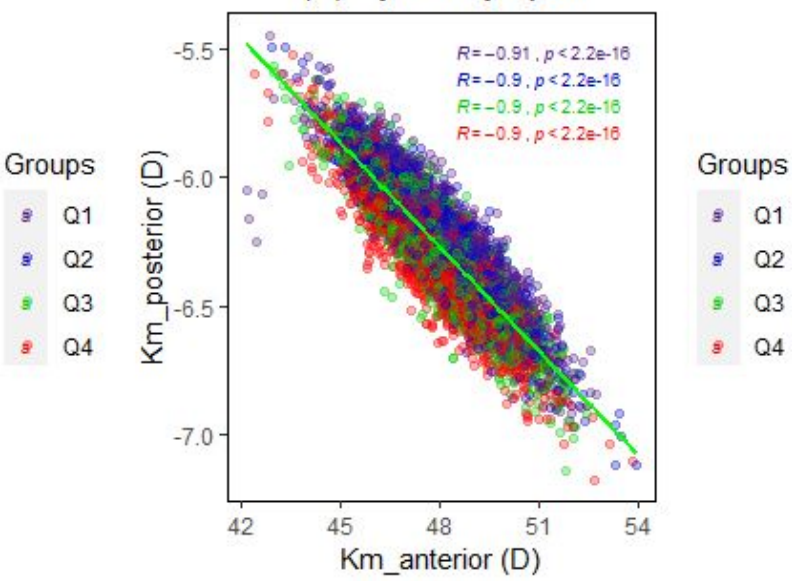

(D) By $\mathrm{ACH}$

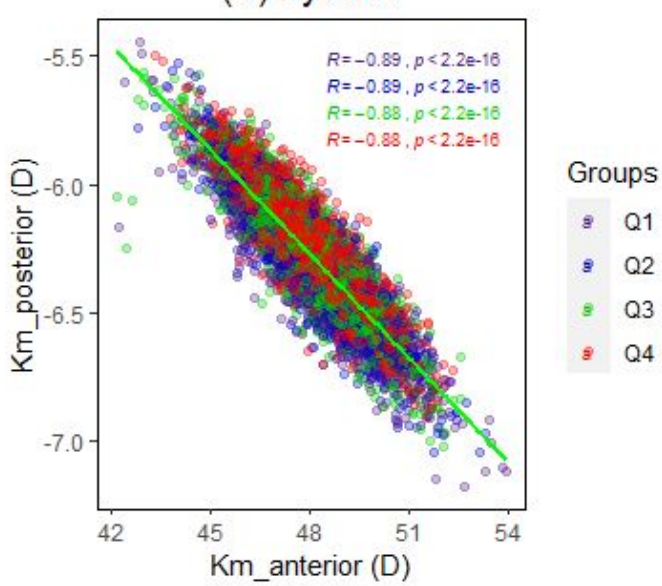

(F) By WTW

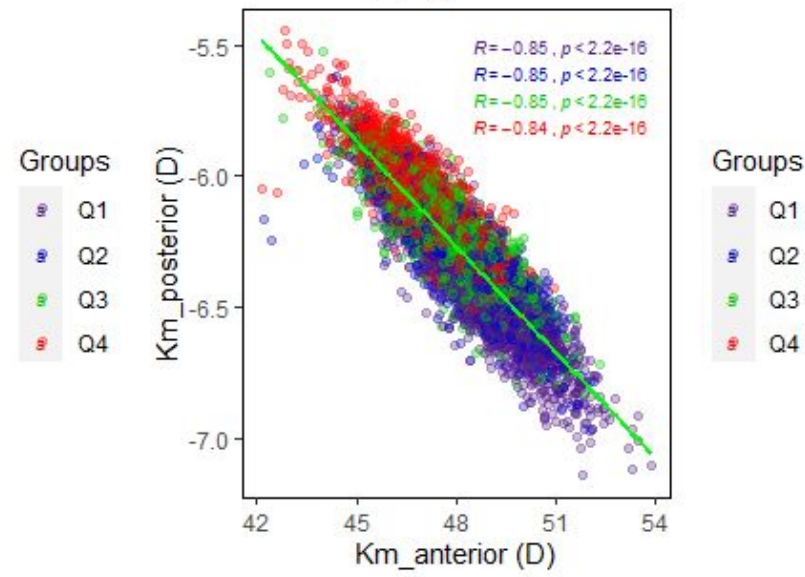

Figure 3

Scattergrams showing anterior-posterior correlations of mean corneal curvature in different quantile groups of SimKm, pachy apex, corneal volume (CV, C), anterior chamber height and volume (ACH and ACV) and white-towhite (WTW) diameter. 
(A) By SimKm

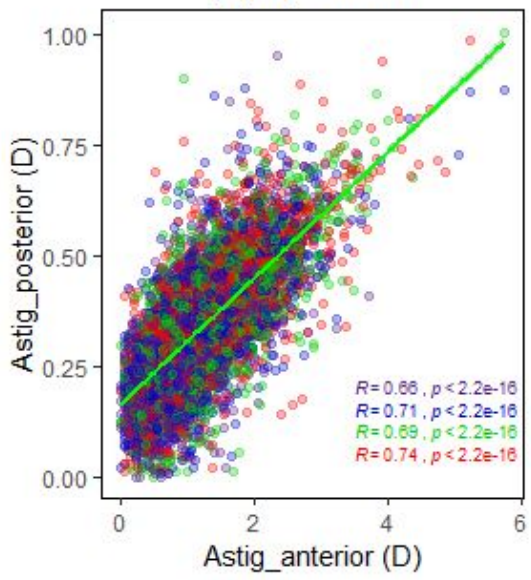

(C) By CV

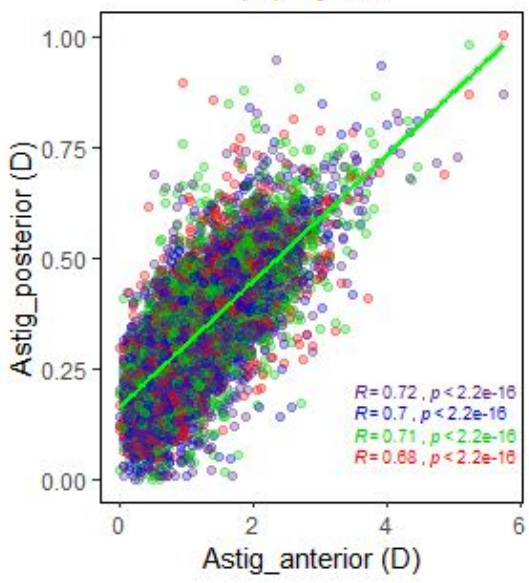

(E) By ACV

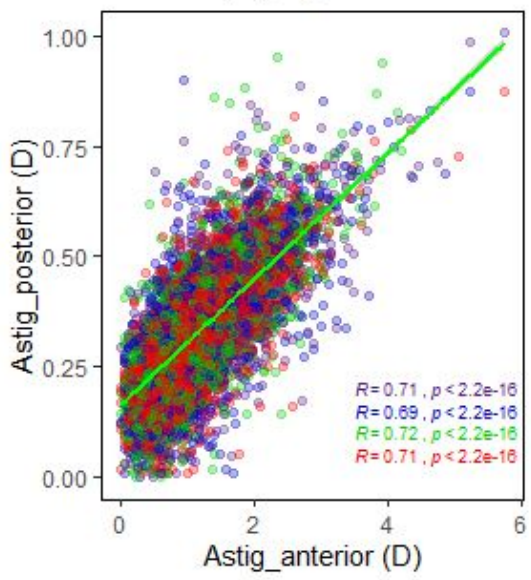

(B) By Pachy apex

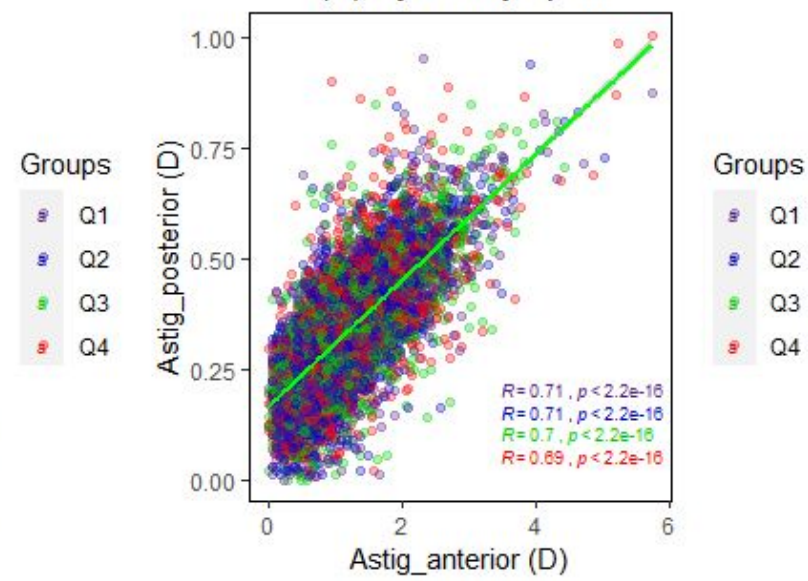

(D) $\mathrm{By} \mathrm{ACH}$

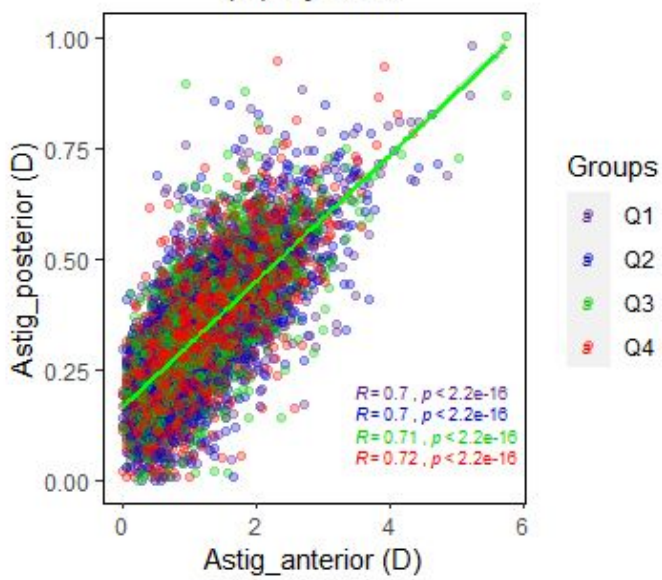

(F) By WTW

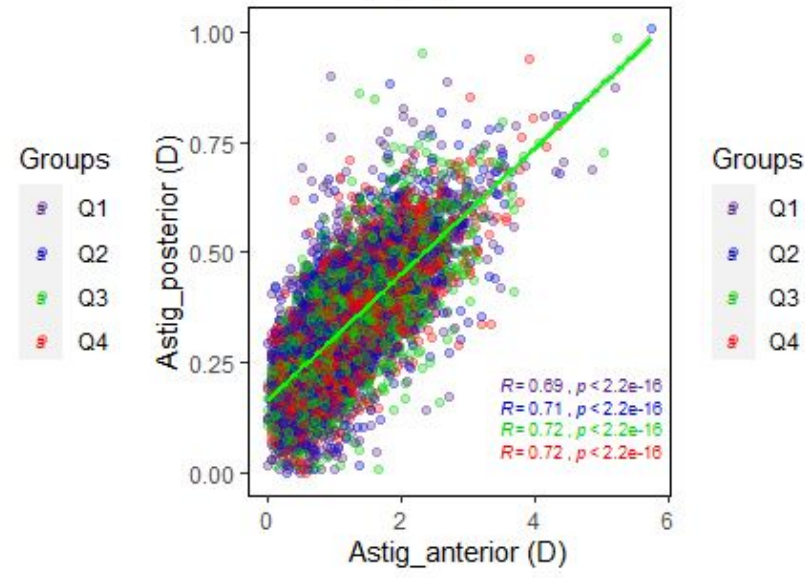

Figure 4

Scattergrams showing anterior-posterior correlations of corneal astigmatism in different quantile groups of SimKm, pachy apex, corneal volume (CV, C), anterior chamber height and volume (ACH and ACV) and white-towhite (WTW) diameter. 
(A) By SimKm

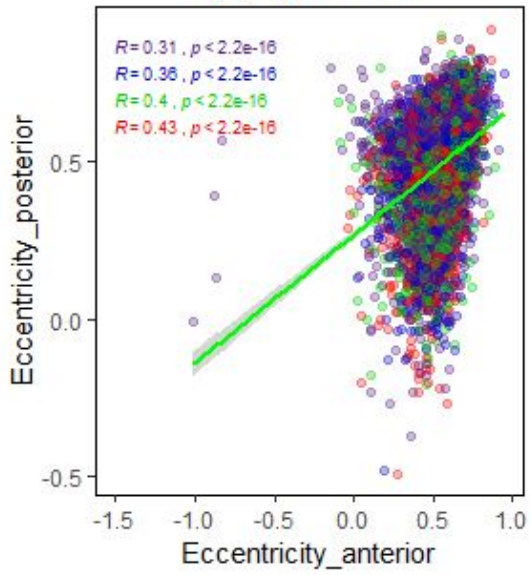

(C) By CV

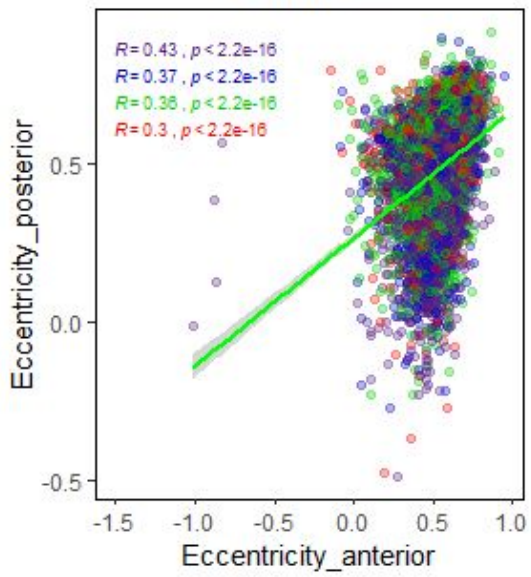

(E) By ACV

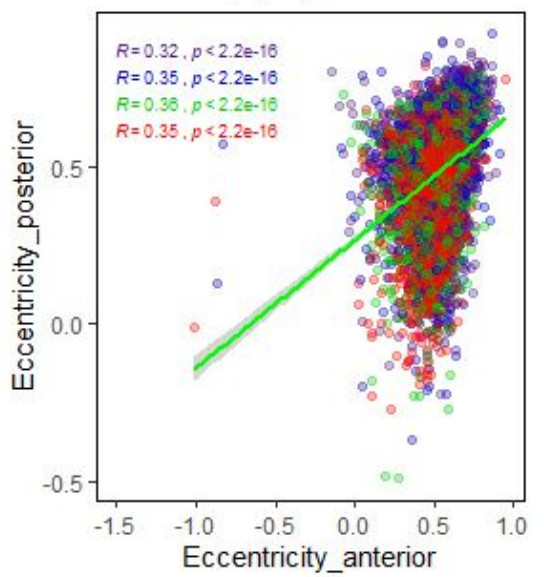

(B) By Pachy apex

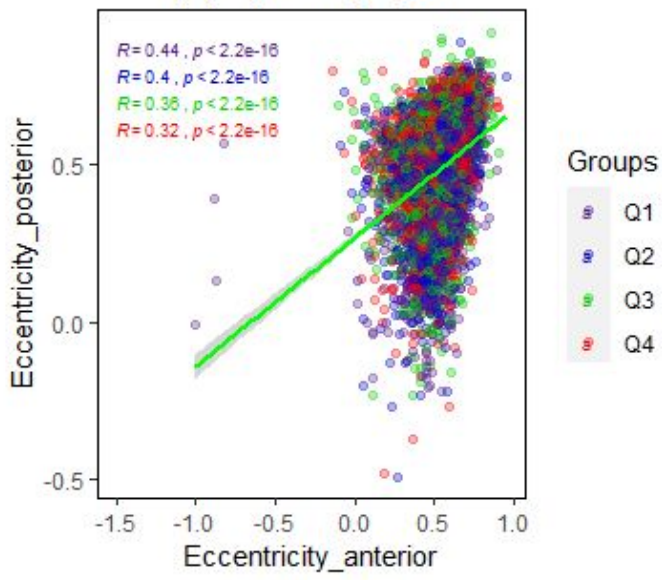

(D) $\mathrm{By} \mathrm{ACH}$

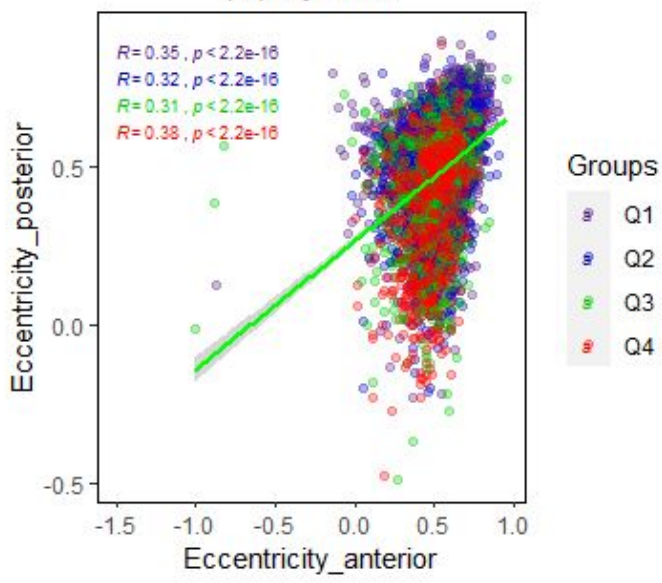

(F) By WTW

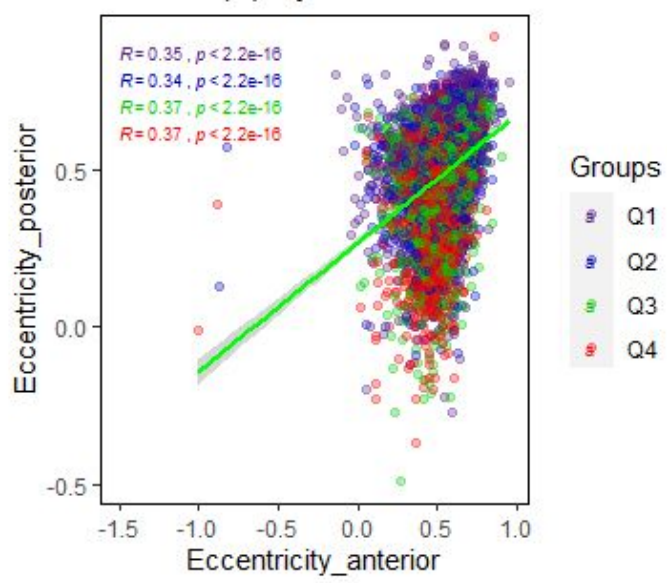

Figure 5

Scattergrams showing anterior-posterior correlations of corneal eccentricity in different quantile groups of SimKm, pachy apex, corneal volume (CV, C), anterior chamber height and volume (ACH and ACV) and white-towhite (WTW) diameter. 
(A) By SimKm

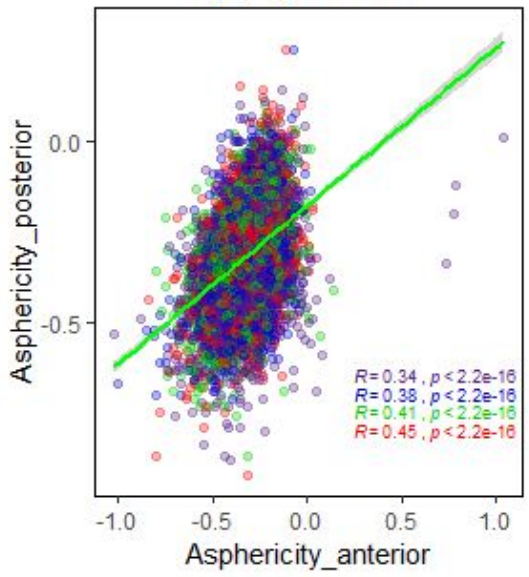

(C) By CV

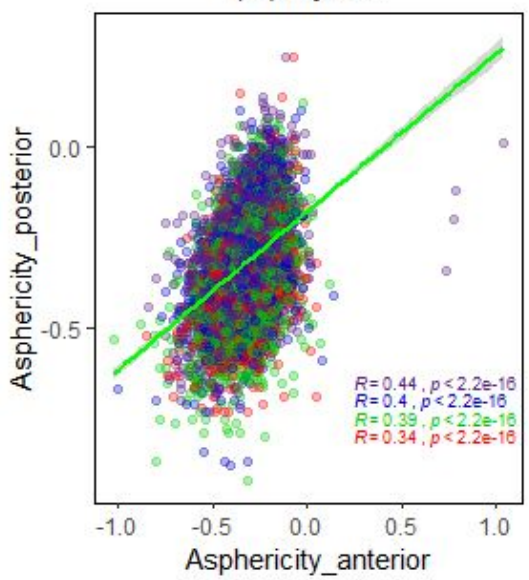

(E) By ACV

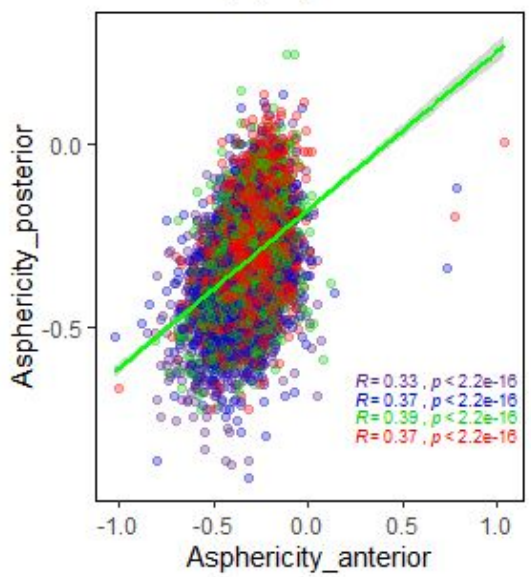

(B) By Pachy apex

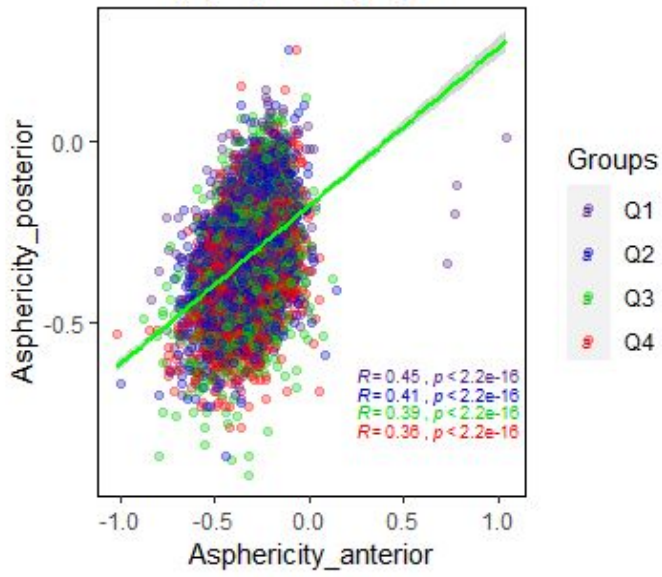

(D) $\mathrm{By} \mathrm{ACH}$

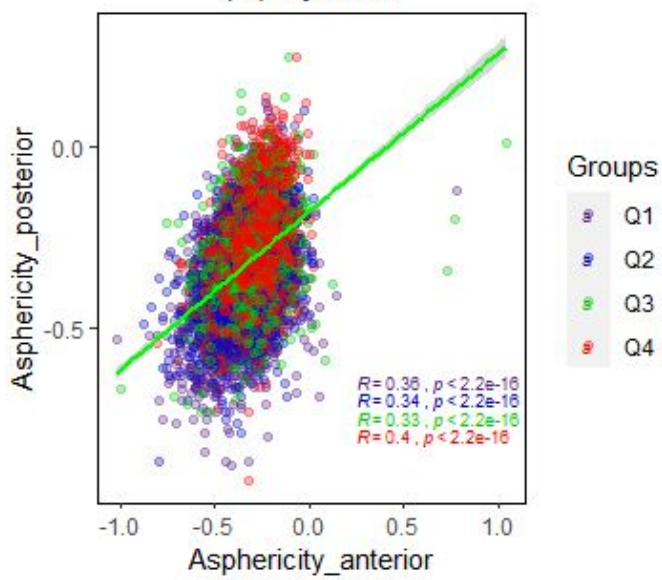

(F) By WTW

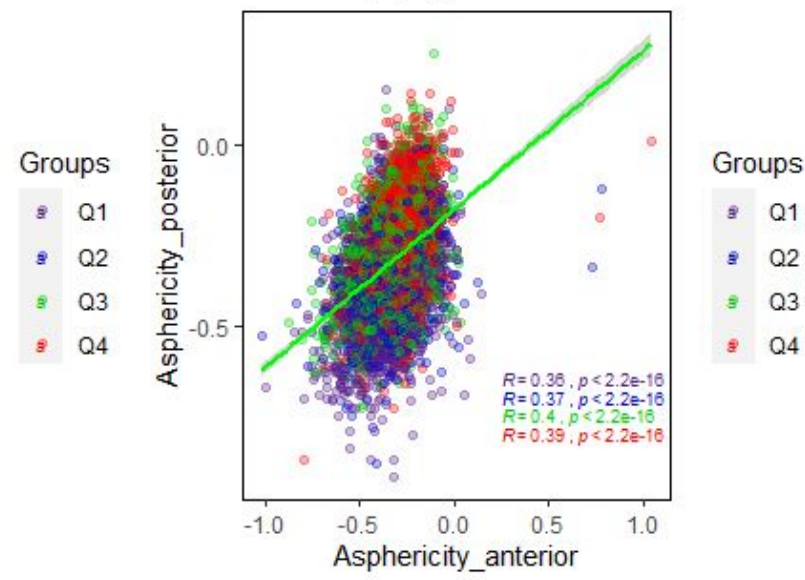

Figure 6

Scattergrams showing anterior-posterior correlations of corneal asphericity in different quantile groups of SimKm, pachy apex, corneal volume (CV, C), anterior chamber height and volume (ACH and ACV) and white-towhite (WTW) diameter. 